\title{
Absolute Magnitude and Kinematics of Barium Stars
}

\section{MARIE-ODILE MENNESSIER ${ }^{1}$, ANA GÓMEZ ${ }^{2}$, XAVIER LURI ${ }^{3}$, SUZANNE GRENIER ${ }^{2}$, LOUIS PRÉVOT ${ }^{4}$, JORDI TORRA ${ }^{3}$, and FRANCESCA FIGUERAS ${ }^{3}$}

${ }^{1}$ Université Montpellier II, Montpellier, France

2 Observatoire de Paris, Meudon, France

${ }^{3}$ Universitat de Barcelona, Barcelona, Spain

4 Observatoire de Marseille, Marseille, France

We apply to $\mathrm{Ba}$ stars a maximum-likelihood method able to distinguish several groups in a given sample and to provide estimates of the mean absolute magnitude and kinematic parameters for each of them. Three group can be distinguished:

- the first contains normal giants,

- the second contains more intrinsically luminous stars,

- the third is smaller and consists of subgiants.

This paper has been published by Gómez et al., $A \& A, 319,881,1997$. 